\title{
Energy using optimization in wireless sensor networks using the hybrid algorithm
}

\author{
SeyedMarouf Hosseini Abbass Ali Rezaei \\ M.A degree, Azad University, Qeshm \\ Seyed.marouf.hosseini@gmail.com \\ Assistant professor, Faculty of computer and information technology, Payame Noor University, Tehran, Iran
}

\begin{abstract}
Energy limitation is one the most important issues in wireless sensor networks design. Data integration and their multi-step and hierarchical transition is an effective method for reducing energy consumption as well as improving energy saving in wireless sensor networks. Network clustering algorithms are significantly more common than the other clustering algorithms due to their reliability and high flexibility. However, in this method, it is needed to appropriately determine the clusters' size and number. In this survey, a data transfer pattern with mixture of multi-step communications and hierarchical transitions has been presented which contains a method for choosing the size and number of clusters according to the synchronous optimization of the in-cluster energy and the energy sent to the main base. The number and size of the clusters in this research would be optimized using genetic optimization algorithm. In the algorithm proposed, first, the environment covered by the sensor nodes which is circular has been divided into various sections and the cluster heads of each section (named local cluster heads) are selected during different send times based on the maximum energy remained among nodes in each section. Hierarchical cluster heads are selected from local cluster heads in a hierarchical cluster according to a specific mechanism based on some parameters including the local cluster head distance from the sink, sum of energy, and the number nodes in each section.
\end{abstract}

Keywords: Sensor networks, hybrid algorithm, genetic optimization, and cluster head

\section{Introduction}

The main issue in designing wireless sensor networks is the sensors' energy limitation. Due to the large number sensors in the networks or the lack of access to them, it is not practical to charge or replace the sensors batteries. Concerning this issue, it is quite necessary to present some approaches regarding the optimization of energy consumption for the network to last longer [4]. Previous studies have shown that it is possible to reach better energy performance through organizing the network sensors [5]. Higher energy efficiency leads the network longevity increase. Establishing a control on the place and number of cluster heads in addition to their size according to the number of members has always been a challenge, thus clustering algorithms efficient in energy consumption and network load moderating are required to solve this problem. The issue of energy and optimizing its utilization is one of the challenges in these networks and numerous actions have been taken about it in the recent years [12-7]. Considering the necessity of the presence of monitoring systems in natural environments (such as jungles and volcanos) and dangerous environments for humans (including nuclear power plants), the existence of wireless sensor networks is highly required, however; the major problem to these networks is their energy consumption and limited capacity of their sensor nodes batteries. Therefore, it is needed to solve the problem of consumption power in order to obtain an acceptable result. Dynamic nature of the problem because of the cluster heads constant changes in each phase of the network performance makes the problem more complicated and causes it to not be modelled by classical mathematical methods. The main goal in this survey is to reduce energy consumption and increase the longevity while maintaining the maximum coverage in wireless sensor networks. It is obvious that the realization of this goal is completely depended on the success rate in secondary objectives. Using reliable optimization algorithms (such as genetic algorithm), it is tried in this thesis to reach the network energy utilization decrease and life time increase by optimizing the effective parameters (the size and number of clusters). Integrating the data sensed from the environment through the cluster heads provides this feasibility to decrease the number of transmitted packets as much as possible. By combining the methods of multi-step and hierarchical sending of the data sensed and reaching a new routing algorithm, it has been tried to use the merits of the both methods in addition to avoiding long-range sending as much as feasible.

\section{Research method}

Nowadays, life cannot be imagined without wireless communications. The advancement of CMOS technology and the creation of small and smaller circuits have made it possible for the wireless circuits to be used in most of today's electronic devices. This advancement has also led to the development of micro sensors. 
These sensors are able to do numerous sensing in some works including voice recognition for the earthquakes and also, they have made it feasible to collect data from isolated places as well as the areas not suitable for human explorations. The name of wireless sensor network is general for various types designed for specific purposes. Analyzing the theses and valid articles available in the field of transferring wireless sensors information to the main base in form of networks as well as delving into the reasoning existed, a method based on coordinates system and reliable optimization algorithms (such as neural networks) has been presented. At the end, validity and efficiency of the presented approach are surveyed using the simulation and then, it is compared with the other available methods. Unlike the conventional networks which are multipurpose, sensor networks are single purpose. Figure (1-1) demonstrates several initial designs of wireless sensor networks.

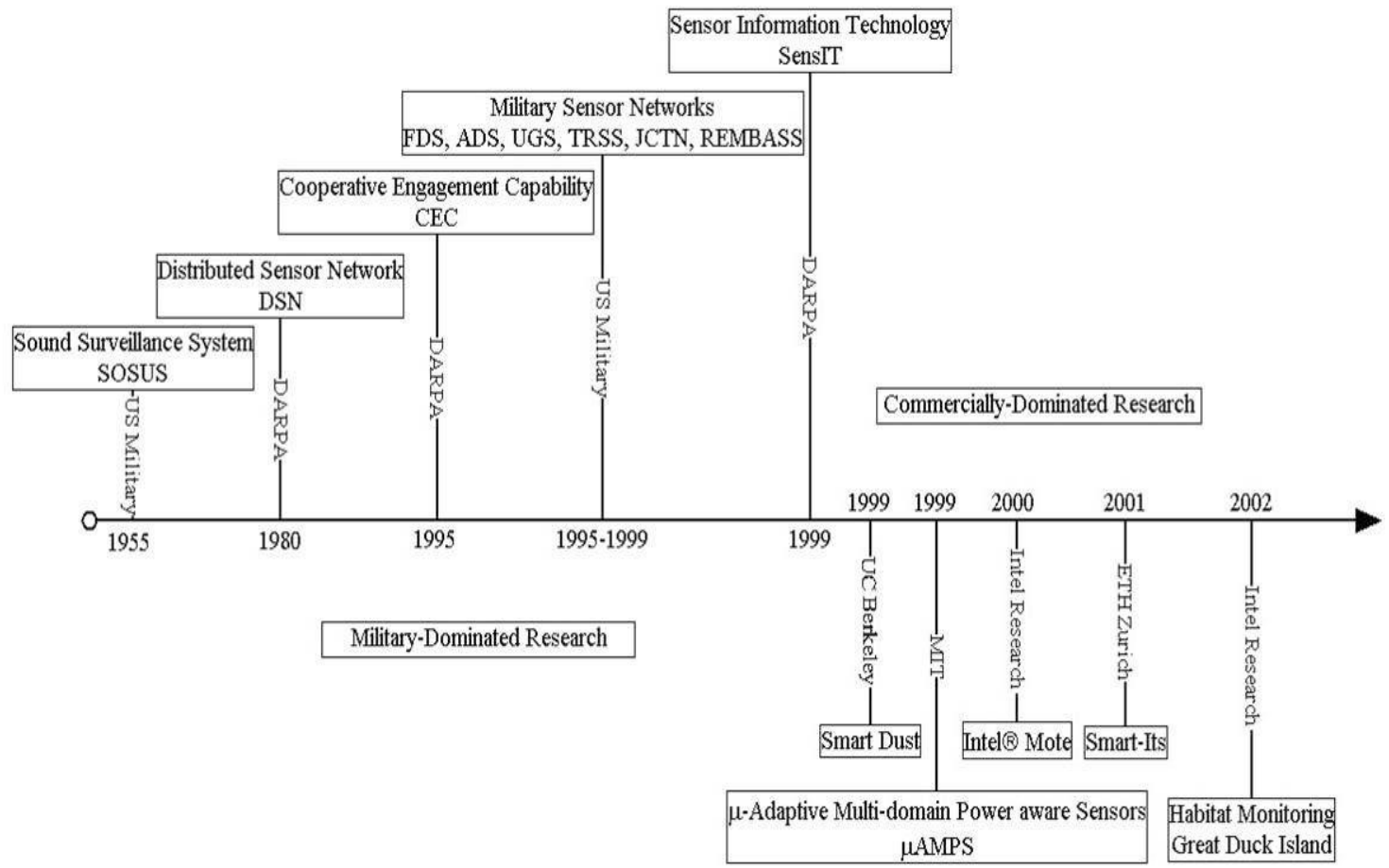

Figure (1-1): The sensor network event logging [13]

Hardware limitations

Topology

Reliability

Scalability

Network longevity increase

Immediate communication and coordination

Security and interferences

Unforeseen factors

The method of data transition based on hierarchical clustering, multi-step sending, and genetic algorithm

First in this method, the optimal size and number of clusters are determined using genetic algorithm. In order to do this, the genetic algorithm is adjusted as the following: the proposed algorithm has two main phases: 1. Clustering phase, 2. Data transition and routing phase.

\section{Clustering phase}

In this stage, first, the entire space under monitoring is divided into concentric circles so as the circles radius difference from each other is equal to the shuck value. Then, the under monitoring area is divided into slack same parts. Each of the areas surrounded by the lines is called a cluster or a section. In fact, the slack and shuck values signify the size and number of each cluster which highly affects the network energy consumption and life time. 


\section{The proposed algorithm efficiency evaluation}

All of the communication lines in this simulation are supposed to have no faults. Presented in the table below are the parameters used in the simulation:

Table (4-1): The simulation parameters

\begin{tabular}{|rr|}
\hline Parameter & Value \\
\hline Number of nodes & 400 \\
Radius of area & $100 \mathrm{~m}$ \\
Location of BS & $(0,0) \mathrm{m}$ \\
Initial energy of each node & $0.5 \mathrm{~J}$ \\
$E_{\text {elec }}$ & $50 \mathrm{~nJ} / \mathrm{bit}$ \\
$\varepsilon_{f s}$ & $0.0013 \mathrm{pJ} /\left(\mathrm{bit} . \mathrm{m}^{4}\right)$ \\
$\varepsilon_{m p}$ & $10 \mathrm{pJ} /\left(\mathrm{bit} . \mathrm{m}^{2}\right)$ \\
$E_{a g s}$ & $5 \mathrm{~nJ} /($ bit.signal $)$ \\
Message size & $4000 \mathrm{bit}$ \\
\hline
\end{tabular}

Genetic algorithm has been used to find the best number of clusters. In order to do this, a simple binary genetic algorithm has been used which its parameters and the intervals assumed for the variables have been depicted in table (4-2).

\begin{tabular}{|ll|}
\hline Parameter & Value \\
\hline Number of population & 20 \\
Number of generations & 200 \\
Crossover probability & 0.75 \\
Mutation probability & 0.05 \\
Shuck size interval & {$[2,100]$} \\
slack number set & $\{1, \ldots, 102\}$ \\
Selection type & Roulette Wheel \\
\hline
\end{tabular}

Table (4-2): The genetic algorithm parameters

After performing genetic algorithm, the best number was chosen as 18 clusters. Figure (4-1) shows the way of optimal clustering. In this part, the suggested algorithm life time specified with the symbol of GAHMEE is compared to S-hierarchical algorithm. As it is clear in the figure, the proposed algorithm longevity is higher than S-hierarchical algorithm. The first nodes death in the suggested and S-hierarchical algorithms happens at the periods of 656 and 371, respectively. The last node in the proposed and S-hierarchical algorithms dies in the periods of 1154 and 1087, respectively. As a result, the suggested algorithm increases the network life time in occurrence of the first and last deaths by $76.81 \%$ and $6.16 \%$, respectively.

\subsection{Comparing with ELHA algorithm}

In this section, the proposed algorithm longevity is compared to ELHA algorithm. As it can be seen in the figure, the proposed algorithm life time is higher than ELHA algorithm. The first node death in the proposed and ELHA algorithms happens in the periods of 656 and 530, respectively. The last node in the proposed algorithm and ELHA algorithm dies in the periods of 1154 and 1080. Thus, the presented algorithm increases the network life time in occurrence of the first and last deaths by $23.77 \%$ and $6.85 \%$, respectively.

Figure (4-6): The number of alive nodes in terms of the sending periods.

Furthermore, the energy consumption in the proposed and ELHA algorithm are compared. Consider figure (4-7), as it can be understood, the proposed algorithm utilizes less energy and has higher energy efficiency in comparison to ELHA algorithm. The rate of load balance for the suggested and ELHA algorithms is 0.43 and 0.47 , respectively. 

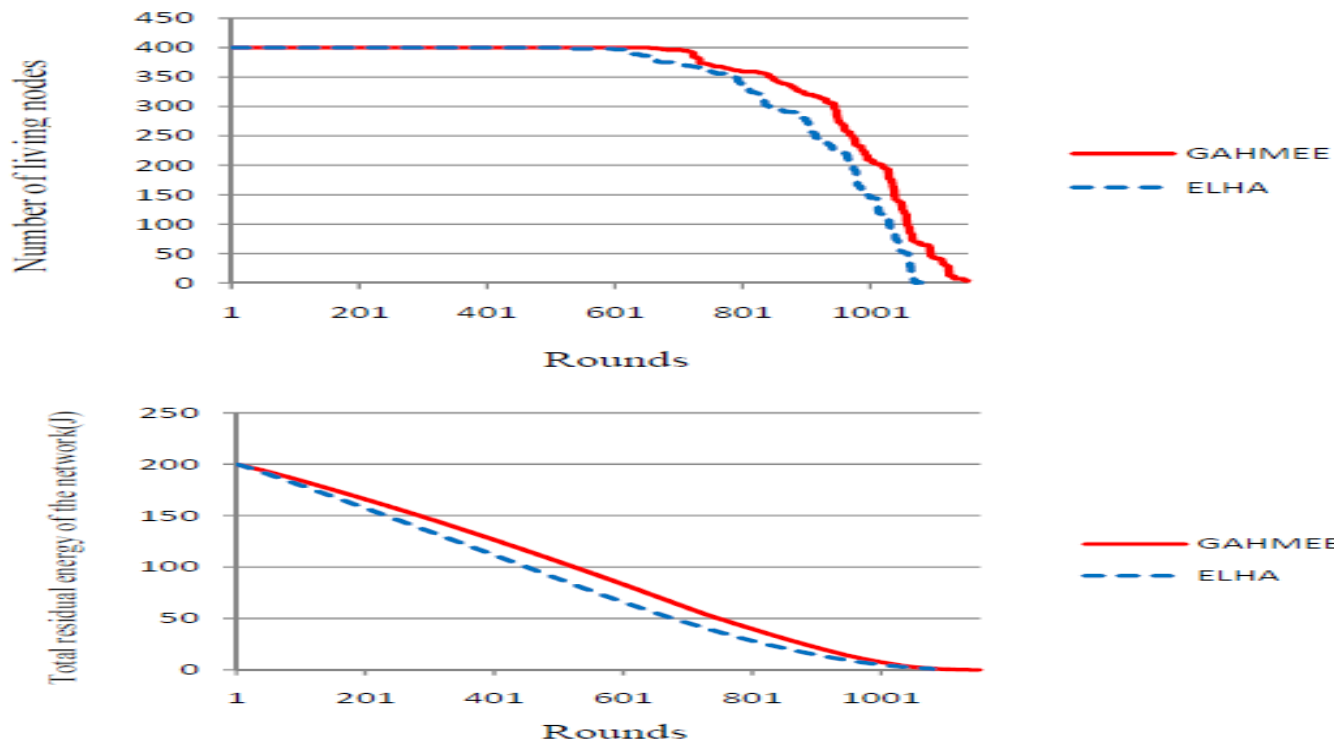

Figure (4-7): The network remaind total energy in terms of the sending periods

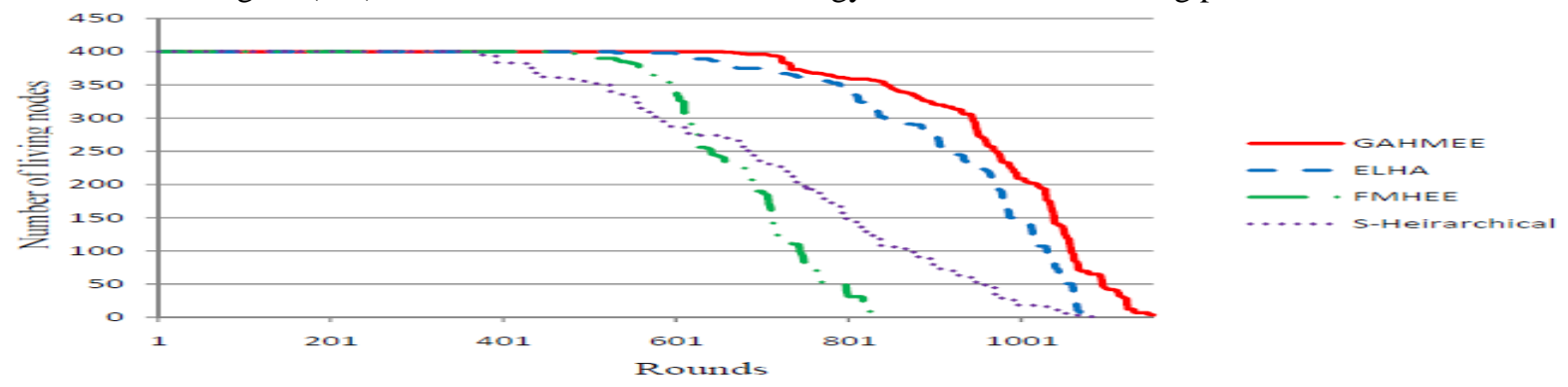

The results of all of the algorithms related to number of alive nodes in the network are presented next to each other in figure (4-8) for the differences to be clearly seen.

Figure (4-8): The number of alive nodes in terms of the sending periods.

Moreover, all of the findings of the algorithms relating to the total energy remained in the network are depicted next to each other in figure (4-9) for the variances to get evident.

Figure (4-9): The network remained total energy in terms of the sending periods.

In the next chapter we will analyze the advantages and disadvantages of the proposed algorithm. The differences between the proposed algorithm and the compared algorithms in terms of the structure and the way of data sending would be stated.

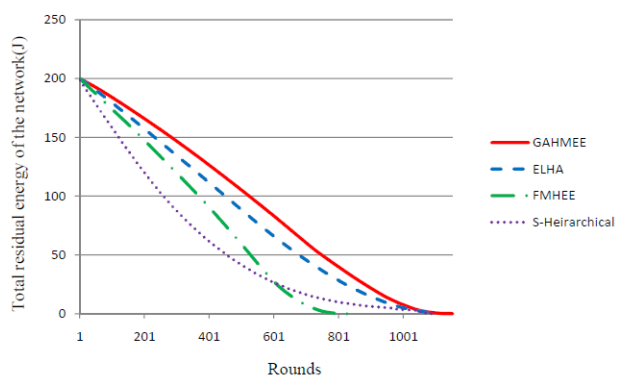

\section{Conclusion}

In the proposed approach, in the network life's last periods, the energy utilization diagram faces slope reductions and the network life continues with very low energy remained in the nodes less chosen as hierarchical cluster heads and close to the sink. In S-hierarchical method, the energy consumption diagram has higher slope at first in comparison to the proposed approach, however, this slope begins to decrease as reaching to the network life time middle periods. In the diagram of the number of alive nodes related to the proposed and ELHA algorithms, the deaths of other nodes happen with higher slope and speed after the death of the first node 
in comparison to other methods which its reason can be stated as the balance in the network energy consumption. In the proposed method which is based on the combination of two methods of multi-step sending and hierarchical sending, other demerits are trimmed using a method so as in order to avoid overloading on the nodes near to the sick in multi-step sending, hierarchical sending method is utilized simultaneously to prevent the energy in the nodes close to the sink to decrease more than its limit. In addition, when the nodes far from the sink are chosen as hierarchical cluster heads in hierarchical method, in order to avoid transitions which are longrange, backward, and opposite to the direction of local cluster heads' sink, multi-step sending is used for these local cluster heads for sending data to the sink which these transitionshave lower range and energy utilization.

\section{References}

[1]. yue J, Zhang W, (2012), Energy Efficient and balanced cluster-based data aggregation algorithm for wireless sensor networks, procedia engineering.

[2]. Hong W.C, Dong, Y, Chen Y, (2012), SVR with hybrid chaotic genetic algorithm for tourism demand forecasting, Journal of applied Soft Computing, page1881-1890.

[3]. Jirong G, Mingcang J, (2010), Housing price forecasting based on genetic algorithm and support vector machine, Journal of Expert Systems.

[4]. Anker T, Bickson D, Dolev D, Hod B, (2008), Efficient clustering for improving network performance in wireless sensor networks, in: Proceedings of the 5th European Conference on Wireless Sensor Networks, March.

[5]. Huang S.C, (2008), Online option price forecasting by using unscented kalman filters and support vector machine, Jornal of Expert Systems.

[6]. Chang B. R, Tsai H.F, (2008), forecast approach using neural network adaption to support vector regression grey model, page 925934.

[7]. Wang T, Yang T.L.X, Zhang D, (2009), An energy efficient and balance hierarchical unequal clustering algorithm for large scale sensor network, Inform. Technol. J. 8 (1), page 28-38.

[8]. Zarandi, M.H.F, Rezaee B, (2009), A 2-type fuzzy rule-based expert system model for stock price analysis, Expert System with Applications, page 139-154.

[9]. Chang P.C, \&liu J, (2008), A TSK type fuzzy rule-based system for stock price prediction, Expert System with Applications,page 135-144.

[10]. Zhuang Y, Pan J, Wu G, (2009), “Energy-optimal grid-based clustering in wireless microsensor networks," IEEE ICDCS Workshop on Wireless Ad hoc and Sensor Networking (WWASN),

[11]. Vidhyapriya R, Vanathi P.T, (2008), "Energy efficient grid-based routing in wireless sensor networks," International Journal of Intelligent Computing and Cybernetics, vol. 1, no. 2, pp. 301-318, Jan.

[12]. Panditharathne C, Sen S.J, (2009), Energy efficient communication protocols for wireless sensor networks, National Institute of Technology Rourkela.

[13]. Liu KH, Cai L, Shen X, (2008), "Exclusive-region based scheduling algorithms for UWB WPAN," IEEE Trans on Wireless Comm.

[14]. Arms S.W, Galbreath J.H, Townsend C.P, Churchill D.L,Corneau B, Ketcham R.P, Phan N, (2009), Energy harvesting wireless sensors and networked timing synchronization for aircraft structural health monitoring, in: Wireless VITAE.

[15]. Hang S.C, chaung P.J, Wu G, (2010), chaos based support vector regression for exchange rate forecasting, Expert System with Applications, page 8590-8598.

[16]. Wang A, Yang D, Sun D, (2012), A clustering algorithm based on energy information and cluster heads expectation for wireless sensor networks, Computers and Electrical Engineering.

[17]. Kim K.J, Han Y, (2008), Genetic algorithm approach to feature discrezation in artifitial neural networks for the prediction of stock price index, Expert System with Applications, page 125-132.

[18]. Qui M, Zhang G.P, (2008), trend time series modeling and forecasting with neural networks, IEEE Transaction on neural networks, page 808-816.

[19]. Anastasi G, Conti M, Francesco M.D, Passarella A, (2009), Energy conservation in wireless sensor networks: a survey, Ad Hoc Networks Journal 7 (3), page 537-568.

[20]. Zhou Z, Xiang X, Wang X, Pan J, (2011), A holistic sensor network design for energy conservation and efficient data dissemination. Computer Networks.

[21]. Lei J, Yates R, Greenstein L, (2009), A generic model for optimizing single hop transmission policy of replenishable sensors, IEEE Transactions on Wireless Communications 8 (2), page 547-551.

[22]. Abdulla A, Nishiyama H, Kato N, (2012), Extending the lifetime of wireless sensor networks: A hybrid routing algorithm, Computer Communications. 